\title{
UNSOLVED LOGISTICAL PROBLEMS IN TIME TRAVEL: SPRING SEMESTER
}

\author{
Your time starts ... now.
}

\section{BY MARISSA LINGEN}

$\mathrm{T}$ The student will offer a 2,000-word laboratory/field write-up solution to one of the following five problems. The student will deliver this solution to the instructor's account by midnight on 20 December, in order to give sufficient time for grading, instructor's subjective timeline. Any student demonstrably and successfully using a Marley device to obtain additional time for this final examination will be given 20 points extra credit on the course grade.

Students with alternative ideas about unsolved logistical problems in time travel should see the instructor for approval of these topics before (relative to their personal timeline) completing an alternate laboratory/field project. Points will be given for clarity, consistency and testability. Students who have not yet (relative to their personal timeline) completed TE1148: Mathematical Approaches to Time Travel Calibration, please note this in your file. All other students must demonstrate temporal consistency mathematically.

1. Cultural exchange with non-human predecessors/antecedents. Taking data on non-human reactions presents a series of challenges from observer effect to simple practicality. Write a plan for presenting a cultural artefact, e.g. Andrew Lloyd Webber's Starlight Express, to a non-human group, e.g. apatosaurs. Take into consideration factors such as the dietary needs of the predecessor/ antecedent, such as apatosaurs' tendency to eat vegetative matter even when it forms a portion of the set.

2. Queueing theory for assassination tourism. If a dozen time travellers show up to assassinate Hitler in the chaos after the Beer Hall Putsch, who gets precedence? How do we fairly and practicably adjust the prioritization factors for era of origin, level of historical plausibility, use of worldline objects and other important factors? Show at least one example.

3. Asymptotic Grandfather Paradox refinements. Although it is well known that the laws of nature (cf. the Novikov self-consistency principle) intervene to keep scholars in the field of temporal dynamics from selfannihilation, exactly how close can one come to these issues? Novel ways of determining proximity are encouraged. Error bars will be extremely important in the report of this field work. There is potential for publication as part of a larger project; please see instructor.

4. Forcing a branch point: parallel universes and the multiplicity of the absurd. Although travel between branch groups has hitherto been impossible (nor do we expect you to change that in a graduate-level course), what trivial changes are large enough to separate off branch groups? Special reference to the work of the Duchamp Theorists of Dada Time Travel is indicated, either to support or deprecate its claims. Students should perform very careful calculations and worldline object non-sentient dry runs to ensure ability to return to a timeline in which this course is held. Credit will be withheld in all timelines in which the course is not held or is taught by a different instructor. Credit will also be withheld from all solutions relying on a Gödel metric. Really, I shouldn't have to say this every term. You should have learned better in TE600.

5. Calculating destinations for small changes outside the main thrust of history. This project will require several examples for comparison and contrast, including early intervention in childhood, early intervention in career, and inspiration of late-period accomplished but obscure scientists, artists and other important figures. Extra credit for producing the kind of small change that ramifies in fields of the professor's particular interest, e.g. extrasolar planetary travel. The instructor does not mind suck-ups as long as they are competent suck-ups.

All travel must take place within the Visser-Roman Ring. Any student violating polygonal symmetry for the purposes of this course will be reported to the Dean of
ONATURE.COM

Follow Futures: @ @ NatureFutures $f$ go.nature.com/mtoodm repercussions includexpulsion. Symmetry is serious business. Students with serious ing possible (likely!)
Please note: Any student wishing to do an experiment falling under the sub-field 'Catalysing Effects of Lesser Dictatorships' will need to fill out Form 753/J12: Experimentation On Human Populations and demonstrate completion (relative to their personal timeline) of TE1120, Ethics Of

Population Shock for Time Travellers. Such experiments must have prior instructor approval and must be accompanied by an instructor or a teaching assistant $a t$ all times.

Students wishing to experiment on their own past selves must fill out Form 753/J15: Waiver of Human Experimentation Protocol Forms. Please bring with you at least three forms of proof that your past self is sufficiently congruent to your current self to qualify. Students wishing to experiment on their own future selves should refer to the Student Counselling Services and obtain from them a Form 753/J27: Waiver of Human Experimentation Protocol Forms - Special Case: Career Counselling.

Office hours are Mondays 10-11 a.m., looped until all student concerns are addressed. If you notice more than four fellow students ahead of you, please bring the instructor a caffeinated beverage, because it's going to be a long day.

Spelling and grammar do matter. Inattention to detail can kill a time traveller. Check your own work. Then check your friends' work. Then check your enemies' work. Check your enemies' work again.

This is a laboratory/field course. All projects must have a travel component not only postulated but accomplished. Although modifications to spatial dampers and other essential equipment are welcome, they are outside the scope of this course, which is meant to be substantially a practicum.

Marissa Lingen has published more than 90 short stories in venues such as Analog, Jim Baen's Universe and Aeon Speculative Fiction. 